\title{
NOTE ON AN ASSOCIATIVE DISTRIBUTIVE ALGEBRA IN WHICH THE COMMUTATIVE LAW OF ADDITION DOES NOT HOLD
}

\author{
BY H. S. VANDIVER
}

1. Introduction. We shall give here a description of an algebra in which the elements are closed under the associative laws of addition and multiplication, a left and a right distributive law holds connecting addition and multiplication, and the usual equality axioms hold, yet the commutative law of addition does not hold.

2. Certain Matrices. As in other papers, ${ }^{*}$ we define a semigroup as a set of elements closed under an associative operation, and a semi-ring as an algebra of the type above described. In the papers just cited we considered a set of elements

$$
C_{1}, C_{2}, \cdots, C_{i}, \cdots, C_{j} ; \quad(j \geqq i),
$$

with $C_{j+1}=C_{i}$ and forming a semi-ring, with

$$
C_{m}+C_{n}=C_{m+n}, \quad C_{m} \cdot C_{n}=C_{m n} .
$$

On page 581 of the first paper mentioned, we showed how to adjoin a zero element to this set, say $C_{0}$.

In (1), set $i=j=1$, and consider the only two distinct elements in the set, after adjoining $C_{0}$. We then have the relations

$$
\begin{aligned}
C_{1}+C_{1}= & C_{1} ; \quad C_{1}+C_{0}=C_{0}+C_{1}=C_{1} ; \quad C_{0}+C_{0}=C_{0} ; \\
& C_{1} \cdot C_{1}=C_{1} ; \quad C_{0} \cdot C_{1}=C_{0} \cdot C_{1}=C_{0} .
\end{aligned}
$$

Note that these elements are not isomorphic to the two residue classes modulo 2, and the cancellation law of addition does not hold in general; that is, from $C_{1}+C_{0}=C_{1}+C_{1}$, we cannot infer $C_{0}=C_{1}$. Consider the matrices

$$
A=\left(\begin{array}{ll}
C_{0} & C_{1} \\
C_{0} & C_{1}
\end{array}\right) ; \quad B=\left(\begin{array}{ll}
C_{1} & C_{0} \\
C_{1} & C_{0}
\end{array}\right) ; \quad C=\left(\begin{array}{ll}
C_{1} & C_{1} \\
C_{1} & C_{1}
\end{array}\right) .
$$

* Proceedings of the National Academy of Sciences, vol. 20 (1934), p. 581; vol. 21 (1935), p. 162. This Bulletin, vol. 40 (1934), pp. 916-920. 
Employing the usual definitions of addition and multiplication of matrices and (2), we have

$$
\begin{gathered}
A+B=C ; \quad A+A=A ; \quad B+B=B ; \\
C+C=C ; \quad A+C=C ; \quad B+C=C . \\
A^{2}=A ; \quad B^{2}=B ; \quad C^{2}=C ; A B=B ; \quad A C=C ; \\
B C=C ; \quad B A=A ; \quad C A=A ; \quad C B=B .
\end{gathered}
$$

We define a left identity in a semi-group $S$ as an element $E_{l}$ such that $E_{l} M=M$ for any $M$ in $S$, with an analogous definition of right identity. An element $O_{r}$ such that $M O_{r}=O_{r}$ for each $M$ in $S$, will be called a right annulator (or annihilator) in $S$, and we define left annulator in an analogous way. If an element is both a right and left annulator it is called an annulator. We note from (3) that $A, B$, and $C$ form a semi-group under addition in which $C$ is an annulator. They form a semi-group under multiplication in which each element is a right-hand annulator and a left-hand identity. By known properties of matrices, since $C_{0}$ and $C_{1}$ form a semi-ring, then $A, B$, and $C$ generate a semi-ring. This result follows also from (3) and (4).

3. Description of the System. Consider a set of bracket symbols $[\alpha, \beta]$, where $\alpha$ and $\beta$ are any of the matrices $A, B$, or $C$. We say that $[\alpha, \beta]=[\gamma, \delta]$ if and only if $\alpha=\gamma, \beta=\delta$. Hence there are nine distinct symbols. Also we define addition and multiplication as follows:

$$
\begin{aligned}
{[\alpha, \beta]+[\gamma, \delta] } & =[\alpha \gamma, \beta+\delta] \\
{[\alpha, \beta] \cdot[\gamma, \delta] } & =[\alpha \gamma, \beta \delta] .
\end{aligned}
$$

From these definitions we note that each element is a right annulator and a left identity under multiplication. Hence the associative law of multiplication and the right and left distributive laws are easily seen to hold. The associative law of addition holds, since

$$
\begin{aligned}
& ([\alpha, \beta]+[\gamma, \delta])+[\epsilon, \theta]=[\alpha \gamma, \beta+\delta]+[\epsilon, \theta] \\
& \quad=[\alpha \gamma \epsilon,(\beta+\delta)+\theta]=[\alpha(\gamma \epsilon), \beta+(\delta+\theta)] \\
& \quad=[\alpha, \beta]+([\gamma, \delta]+[\epsilon, \theta]) .
\end{aligned}
$$


Hence the bracket symbols form a semi-ring. The commutative law of addition does not hold in general in this semi-ring since

$$
\begin{aligned}
& {[A, \beta]+[B, \gamma]=[A B, \beta+\gamma],} \\
& {[B, \gamma]+[A, \beta]=[B A, \gamma+\beta],}
\end{aligned}
$$

but $B A \neq A B$. These symbols have a property under addition which might be called quasi-commutativity:

$$
\begin{aligned}
{[\alpha, \beta]+[\alpha, \beta]+[\gamma, \delta]+} & {[\gamma, \delta] } \\
& =[\alpha, \beta]+[\gamma, \delta]+[\alpha, \beta]+[\gamma, \delta],
\end{aligned}
$$

for the left-hand member reduces to $[\gamma, \beta+\beta+\delta+\delta]$ and the right to $[\gamma, \beta+\delta+\beta+\delta]$, which are equal since $A, B$, and $C$ are commutative under addition. It is also easy to see that $M N M N=M M N N$, for $M$ and $N$ are bracket symbols.

The University of Texas

\section{BRANCHED AND FOLDED COVERINGS*}

BY A. W. TUCKER

A simple example of a branched covering arises when one sphere is mapped on another so that each point of the first sphere goes into the point of the second which has the same latitude but double the longitude. This is a covering of degree two with simple branching at the north and south poles. As an example of a folded covering we take a torus, thought of as a sphere with a handle on one side, and project it radially inward on a smaller concentric sphere. The torus covers the sphere once but with a fold produced by collapse of the handle. The product of this torus-sphere covering with the previous spheresphere covering yields a torus-sphere covering of degree two which is both branched and folded. Suitable triangulation of the torus and the spheres will turn the above mappings into simplicial mappings in which each simplex maps barycentrically into a simplex of the same dimension. In what follows we make some rudimentary calculations concerning the branching and folding of a simplicial covering of one $n$-dimensional complex by

\footnotetext{
* Presented to the Society, September 1, 1936.
} 